\title{
BLOW-UP SOLUTIONS FOR LINEAR PERTURBATIONS OF THE YAMABE EQUATION
}

\author{
PIERPAOLO ESPOSITO, ANGELA PISTOIA, AND JÉRÔME VÉTOIS
}

\begin{abstract}
For a smooth, compact Riemannian manifold $(M, g)$ of dimension $N \geq 3$, we are interested in the critical equation

$$
\Delta_{g} u+\left(\frac{N-2}{4(N-1)} S_{g}+\varepsilon h\right) u=u^{\frac{N+2}{N-2}} \quad \text { in } M, \quad u>0 \quad \text { in } M,
$$

where $\Delta_{g}$ is the Laplace-Beltrami operator, $S_{g}$ is the Scalar curvature of $(M, g), h \in C^{0, \alpha}(M)$, and $\varepsilon$ is a small parameter.
\end{abstract}

\section{INTRODUCTION}

Letting $(M, g)$ be a smooth, compact Riemannian $N$-manifold, $N \geq 3$, we consider the solutions $u \in C^{2, \alpha}$ of the problem

$$
\Delta_{g} u+\kappa u=c u^{p}, \quad u>0 \quad \text { in } M,
$$

where $\Delta_{g}:=-\operatorname{div}_{g} \nabla$ is the Laplace-Beltrami operator, $\kappa \in C^{0, \alpha}(M), \alpha \in(0,1), c \in \mathbb{R}$, and $p>1$.

When $\kappa=\alpha_{N} S_{g}$ and $p=2^{*}-1$, where $\alpha_{N}:=\frac{N-2}{4(N-1)}, S_{g}$ is the Scalar curvature of $(M, g)$ and $2^{*}:=\frac{2 N}{N-2}$ is the critical Sobolev exponent, equation (1.1) reads as

$$
\Delta_{g} u+\frac{N-2}{4(N-1)} S_{g} u=c u^{\frac{N+2}{N-2}}, \quad u>0 \quad \text { in } M,
$$

and is referred to in the literature as the Yamabe problem. The constant $c$ can be restricted to the values $-1 / 1$ or 0 depending on whether the Yamabe invariant of $(M, g)$, namely

$$
\mu_{g}(M)=\inf _{\widetilde{g} \in[g]}\left(\operatorname{Vol}_{\widetilde{g}}(M)^{\frac{2-N}{N}} \int_{M} S_{\widetilde{g}} d v_{\widetilde{g}}\right)
$$

has negative/positive sign or vanishes, respectively, where $[g]=\left\{\phi g: \phi \in C^{\infty}(M), \phi>0\right\}$ is the conformal class of $g$ and $\operatorname{Vol}_{\widetilde{g}}(M)$ is the volume of the manifold $(M, \widetilde{g})$. If $u$ is a solution of (1.2), then the metric $\widetilde{g}=u^{4 /(N-2)} g$ has constant Scalar curvature and belongs to $[g]$.

The Yamabe problem, raised by H. Yamabe [42] in '60, was firstly solved by Trudinger [41] when $\mu_{g}(M) \leq 0$. In this case, the solution is unique (up to a normalization when $\mu_{g}(M)=0$ ). In general, a solution of (1.2) can be found by a direct constrained minimization method. As shown by Aubin [1], the inequality

$$
\mu_{g}(M)<\mu_{g_{0}}\left(\mathbb{S}^{N}\right)
$$

where $\left(\mathbb{S}^{N}, g_{0}\right)$ is the round sphere, is the key ingredient to show compactness of minimizing sequences, a non-trivial fact in view of the non-compactness of the Sobolev embedding $H_{1}^{2}(M) \hookrightarrow L^{2^{*}}(M) . \quad\left(\mathbb{S}^{N}, g_{0}\right)$ has already constant Scalar curvature. For manifolds $(M, g)$

Date: October 1st, 2012. 
which are not conformally equivalent to $\left(\mathbb{S}^{N}, g_{0}\right)\left((M, g) \neq\left(\mathbb{S}^{N}, g_{0}\right)\right.$ for short) with $\mu_{g}(M)>0$, the Yamabe equation (1.2) has been solved via (1.3) by:

- Aubin [1] in the non-locally conformally flat case with $N \geq 6$, by exploiting the nonvanishing of the Weyl curvature tensor Weyl $_{g}$ of $(M, g)$ in the construction of local test functions;

- Schoen [37] when either $N=3,4,5$ or $(M, g) \neq\left(\mathbb{S}^{N}, g_{0}\right)$ is locally conformally flat, by exploiting the Positive Mass Theorem by Schoen-Yau [39, 40] in the construction of global test functions

(see also Lee-Parker [22] for a unified approach).

From now on, we restrict our attention to the case where $(M, g)$ has positive Yamabe invariant $\mu_{g}(M)>0$. When $(M, g) \neq\left(\mathbb{S}^{N}, g_{0}\right)$, Schoen [38] addressed the question of the compactness of Yamabe metrics, and he proved the compactness to be true in the locally conformally flat case [38]. Recently, compactness of Yamabe metrics has been proved to be true for a general manifold $(M, g) \neq\left(\mathbb{S}^{N}, g_{0}\right)$ of dimension $N \leq 24$ by Khuri-Marques-Schoen [21]. Unexpectedly, compactness of Yamabe metrics has revealed to be false in general in dimensions $N \geq 25$ by Brendle [5] and Brendle-Marques [6]. Previous contributions where the compactness of Yamabe metrics is proved in lower dimensions are by Li-Zhu [27] $(N=3)$, Druet [10] $(N \leq 5)$, Marques [28] $(N \leq 7)$, and Li-Zhang [24 26] $(N \leq 11)$. In all these results, it is shown that sequences of solutions $\left(u_{k}\right)_{k \in \mathbb{N}}$ of (1.1) with $\kappa \equiv \alpha_{N} S_{g}, c=1$, and exponents $\left(p_{k}\right)_{k \in \mathbb{N}}$ in $\left[1+\varepsilon_{0}, 2^{*}-1\right], \varepsilon_{0}>0$ fixed, are pre-compact in $C^{2, \alpha}(M), \alpha \in(0,1)$.

When $\kappa \not \equiv \alpha_{N} S_{g}$, the situation is different. When $\kappa<\alpha_{N} S_{g}$, Druet [9, 10] (see also DruetHebey 13 and Druet-Hebey-Vétois 16]) proved that compactness does hold for equation (1.1) with $c=1$ and exponents $p$ in the range $\left[1+\varepsilon_{0}, 2^{*}-1\right]$, for all dimensions $N \geq 3$ (in case $N=3$, it is possible to write a more refined condition on the mass, see Li-Zhu [27]). As shown in Micheletti-Pistoia-Vétois [29] and Pistoia-Vétois [32], in dimensions $N \geq 4$, such a compactness result does not hold when $\kappa\left(\xi_{0}\right)>\alpha_{n} S_{g}\left(\xi_{0}\right)$ at some point $\xi_{0} \in M$ with a nondegeneracy assumption at $\xi_{0}$, and, see [29], compactness does not hold either in the supercritical range $p>2^{*}-1$ when $\kappa\left(\xi_{0}\right)<\alpha_{N} S_{g}\left(\xi_{0}\right)$ at some point $\xi_{0} \in M$. We also refer to Robert-Vétois [36, Theorem 2.3] where a special non-compactness result is obtained in dimension $N=6$ for potentials $\kappa>\alpha_{N} S_{g}$ (see also Druet [9] and Druet-Hebey [11, 12] in case of $(M, g)=\left(\mathbb{S}^{N}, g_{0}\right)$ with $\left.N=6\right)$. In the locally conformally flat case with $N \geq 4$, Hebey-Vaugon [19] proved that there always exists $\widetilde{g} \in[g]$ such that the equation $\Delta_{\widetilde{g}} u+$ $\alpha_{N} \max _{M}\left(S_{\widetilde{g}}\right) u=u^{2^{*}-1}$ in $M$ is not compact. In case $(M, g)=\left(\mathbb{S}^{N}, g_{0}\right)$ with $N \geq 5$ and when $\left(\kappa-\alpha_{N} S_{g}\right)$ is a positive constant, Chen-Wei-Yan [8] proved that equation (1.1) with $c=1$ and $p=2^{*}-1$ is not compact (see also the constructions by Hebey-Wei [20] in case $N=3$ ).

When the potential $\kappa$ varies, for manifolds $(M, g) \neq\left(\mathbb{S}^{N}, g_{0}\right)$ with $\mu_{g}(M)>0$, Druet [10] (see also Druet-Hebey [14]) proved that sequences of solutions $\left(u_{k}\right)_{k \in \mathbb{N}}$ of (1.1) with $c=1$, exponents $\left(p_{k}\right)_{k \in \mathbb{N}}$ in $\left[1+\varepsilon_{0}, 2^{*}-1\right]$, and potentials $\left(\kappa_{k}\right)_{k \in \mathbb{N}}$, are pre-compact in $C^{2, \alpha}(M)$, $\alpha \in(0,1)$, when $n=3,4,5$ provided that $\kappa_{k} \leq \alpha_{n} S_{g}$. The same result is strongly expected to be true in the locally conformally flat case and generally for $N \leq 24$.

The aim of the paper is to investigate the effect of positive perturbations of the geometric potential by exhibiting the failure of compactness properties for the equation

$$
\Delta_{g} u+\left(\alpha_{N} S_{g}+\varepsilon h\right) u=u^{2^{*}-1}, \quad u>0 \quad \text { in } M,
$$

where $h \in C^{0, \alpha}(M), \alpha \in(0,1)$, with $\max _{M} h>0$ and $\varepsilon>0$ is a small parameter. 
A family $\left(u_{\varepsilon}\right)_{\varepsilon}$ of solutions to equation (1.4) is said to blow up at some point $\xi_{0} \in M$ if there holds $\sup _{U} u_{\varepsilon} \rightarrow+\infty$ as $\varepsilon \rightarrow 0$, for all neighborhoods $U$ of $\xi_{0}$ in $M$. Letting

$$
E(\xi):=\frac{h(\xi)}{\left|\operatorname{Weyl}_{g}(\xi)\right|_{g}},
$$

our main result is:

Theorem 1.1. Let $(M, g) \neq\left(\mathbb{S}^{N}, g_{0}\right)$ be a smooth, compact, non-locally conformally flat Riemannian manifold with $N \geq 6$ and $\mu_{g}(M)>0$. Let $h \in C^{0, \alpha}(M), \alpha \in(0,1)$, so that $\max _{M} h>0$ and $\inf \left\{\left|\operatorname{Weyl}_{g}(x)\right|_{g}: h(x)>0\right\}>0$. Then for $\varepsilon>0$ small, equation (1.4) has a solution $u_{\varepsilon}$ such that the family $\left(u_{\varepsilon}\right)_{\varepsilon}$ blows up, up to a sub-sequence, as $\varepsilon \rightarrow 0$ at some point $\xi_{0}$ so that $E\left(\xi_{0}\right)=\max _{M} E$.

Introducing the "reduced energy" $\widetilde{E}:(0, \infty) \times M \rightarrow \mathbb{R}$ defined as

$$
\widetilde{E}(d, \xi)=c_{2} d^{2} h(\xi)-c_{3} d^{4}\left|\operatorname{Weyl}_{g}(\xi)\right|_{g}^{2}
$$

with $c_{2}, c_{3}>0$, Theorem 1.1 is an easy consequence of the following more general result:

Theorem 1.2. Let $(M, g) \neq\left(\mathbb{S}^{N}, g_{0}\right)$ be a smooth, compact, non-locally conformally flat Riemannian manifold with $N \geq 6$ and $\mu_{g}(M)>0$, and $h \in C^{0, \alpha}(M), \alpha \in(0,1)$. Assume that there exists a $C^{0}$-stable critical set $\mathcal{D} \subset(0, \infty) \times M$ of $\widetilde{E}$. Then for $\varepsilon>0$ small, equation (1.4) has a solution $u_{\varepsilon}$ such that the family $\left(u_{\varepsilon}\right)_{\varepsilon}$ blows up, up to a sub-sequence, at some $\xi_{0} \in \pi(\mathcal{D})$, where $\pi:(0, \infty) \times M \rightarrow M$ is the projection operator onto the second component.

According to $\mathrm{Li}[23$, we say that a compact set $\mathcal{D} \subset(0, \infty) \times M$ of critical points of $\widetilde{E}$ is a $C^{0}$-stable critical set of $\widetilde{E}$ if for any compact neighborhood $U$ of $\mathcal{D}$ in $(0, \infty) \times M$, there exists $\delta>0$ such that, if $\mathcal{J} \in C^{1}(U)$ and $\|\mathcal{J}-\widetilde{E}\|_{C^{0}(U)} \leq \delta$, then $\mathcal{J}$ has at least one critical point in $U$.

Given $\xi \in M$ so that $h(\xi)>0$, define $d(\xi)$ as

$$
d(\xi)=\left(\frac{c_{2} h(\xi)}{2 c_{3} \mid \text { Weyl }\left._{g}(\xi)\right|_{g} ^{2}}\right)^{1 / 2}
$$

with the convention that $d(\xi)=+\infty$ if $\operatorname{Weyl}_{g}(\xi)=0$. Given $\xi \in M$ with $h(\xi)>0$, the function $\widetilde{E}$ is increasing for $d \in(0, d(\xi))$ and, if $d(\xi)<+\infty$, achieves its global maximum in $d$ at $d(\xi)$. Since

$$
\widetilde{E}(d(\xi), \xi)=\frac{c_{2}^{2} h^{2}(\xi)}{4 c_{3}\left|\mathrm{Weyl}_{g}(\xi)\right|_{g}^{2}}=\frac{c_{2}^{2}}{4 c_{3}} E(\xi)^{2},
$$

in order to derive Theorem 1.1 , the set $\mathcal{D}$ in Theorem 1.2 is constructed as

$$
\mathcal{D}=\left\{(d(\xi), \xi): \xi \in M \text { s.t. } E(\xi)=\max _{M} E\right\},
$$

which is clearly a $C^{0}$-stable critical set of $\widetilde{E}$. Since $d(\xi)$ is a maximum point of $\widetilde{E}$ in $d$, neither minimum points of $E$, nor saddle points of $E$ can provide any $C^{0}$ stable critical set of $\widetilde{E}$.

Let us finally compare problem (1.4) with its Euclidean counter-part on a smooth bounded domain $\Omega \subset \mathbb{R}^{N}, N \geq 4$, with homogeneous Dirichlet boundary condition:

$$
\Delta_{\text {Eucl }} u+\lambda u=u^{2^{*}-1} \text { in } \Omega, \quad u>0 \text { in } \Omega, \quad u=\text { on } \partial \Omega \text {. }
$$


For $\lambda \geq 0$, a direct minimization method (for the corresponding Rayleigh quotient) never gives rise to any solution of (1.5), and no solutions exist at all if $\Omega$ is star-shaped as shown by Pohožaev [33]. Moreover, following the arguments developed by Ben Ayed-El MehdiGrossi-Rey [3], problem (1.5) has never any solution with a single blow-up point as $\lambda \rightarrow 0^{+}$. The effect of the geometry, which is crucial to provide a solution for the Yamabe problem (corresponding to $\lambda=0$ in (1.5) ) by minimization, is also relevant to producing solutions of (1.4) (corresponding to $\lambda \rightarrow 0^{+}$in (1.5) ) with a single blow-up point as stated in Theorem 1.1. When $\lambda<0$, solutions of (1.5) can be found by direct minimization as shown by BrezisNirenberg [7, and exhibit a single blow-up point as $\lambda \rightarrow 0^{-}$as shown by Han [18, in contrast with the compactness property proved by Druet [9, 10]. Solutions of (1.5) with a single blow-up point, see Rey [34, 35], and with multiple blow-up points, see Bahri-Li-Rey [2] and MussoPistoia [30], as $\lambda \rightarrow 0^{-}$have been constructed in a very general way.

We attack the existence issue of blowing-up solutions by a perturbative method, referred to in the literature as the non-linear Lyapunov-Schmidt reduction. Such a method is well known and the main point is to produce a suitable ansatz for the solutions. In the non-locally conformally flat case with $N \geq 6$ the basic ansatz is like in Aubin [1, but, see Section 2 , needs to be slightly corrected via linearization so to account for the local geometry. A similar idea has been used for the prescribed $Q$-curvature problem by Pistoia-Vaira [31, the fourthorder analogue of the Yamabe problem. An alternative and more geometrical approach can be devised based on the conformal covariance of $\Delta_{g}+\alpha_{N} S_{g}$. The main point is to allow the metric $g$ to vary in the conformal class so to gain flatness at each point $\xi \in M$, and this approach allows us, see Esposito-Pistoia-Vétois [17, to cover in an unified way also the remaining cases $N=4,5$ or $(M, g)$ locally conformally flat with $N \geq 6$ (the case $N=3$ is always excluded by the compactness result of $\mathrm{Li}-\mathrm{Zhu}$ [27]). The aim of this paper is at the same time to advertise the general result contained in [17, and to provide a simpler and more intuitive proof in a special case. Thanks to the solvability theory of the linearized operator, we are led to study critical points of a finite-dimensional functional $\mathcal{J}_{\varepsilon}$, and a key step is to obtain in Section 3 an asymptotic expansion of $\mathcal{J}_{\varepsilon}$ by identifying the "reduced energy" $\widetilde{E}$ as the main order term. In Section 4, we describe the main steps of the non-linear Lyapunov-Schmidt reduction, and we deduce our general result Theorem 1.2 .

\section{The CORRECTING TERM TOWARDS AN IMPROVED ANSATZ}

Letting

$$
U(r)=\left(\frac{\sqrt{N(N-2)}}{1+r^{2}}\right)^{\frac{N-2}{2}}
$$

we aim to solve

$$
\Delta V+p U^{p-1} V=\frac{1}{3} \sum_{i, j=1}^{N} R_{i j}(\xi) \frac{y^{i} y^{j}}{|y|} \partial_{r} U+\alpha_{N} S_{g}(\xi) U,
$$

where $p=\frac{N+2}{N-2}$ and $R_{i j}$ are the components of the Ricci tensor $\operatorname{Ric}_{g}$ of $(M, g)$ in geodesic coordinates. Here, $\Delta=\sum_{i=1}^{N} \frac{\partial^{2}}{\partial y_{i}^{2}}$ is the Euclidean laplacian with the standard sign convention, and $U(|y|)$ is the unique positive radial solution of $-\Delta U=U^{p}$ with $U(0)=\max _{\mathbb{R}^{N}} U=[N(N-$ 2) $]^{\frac{N-2}{4}}$. 
Since $S_{g}(\xi)=\sum_{i=1}^{N} R_{i i}(\xi)$, a straightforward computation shows that

$$
V(y)=[N(N-2)]^{\frac{N-2}{4}}\left(\frac{|y|^{2}+3}{12\left(1+|y|^{2}\right)^{\frac{N}{2}}} \sum_{i, j=1}^{N} R_{i j}(\xi) y^{i} y^{j}-\frac{S_{g}(\xi)}{24(N-1)} \frac{|y|^{4}+3}{\left(1+|y|^{2}\right)^{\frac{N}{2}}}\right)
$$

is a solution of (2.2) as we were searching for.

Let $0<r_{0}<i_{g}(M)$, where $i_{g}(M)$ is the injectivity radius of $(M, g)$. Take $\chi$ a smooth cutoff function such that $0 \leq \chi \leq 1$ in $\mathbb{R}, \chi \equiv 1$ in $\left[-r_{0} / 2, r_{0} / 2\right]$, and $\chi \equiv 0$ out of $\left[-r_{0}, r_{0}\right]$. For any point $\xi$ in $M$ and for any positive real number $\mu$, we define the functions $\mathcal{U}_{\mu, \xi}$ and $\mathcal{V}_{\mu, \xi}$ on $M$ by

$$
\mathcal{U}_{\mu, \xi}(z)=\chi\left(d_{g}(z, \xi)\right) U_{\mu}\left(d_{g}(z, \xi)\right), \mathcal{V}_{\mu, \xi}(z)=\chi\left(d_{g}(z, \xi)\right) V_{\mu}\left(\exp _{\xi}^{-1}(z)\right),
$$

where $d_{g}$ is the geodesic distance in $(M, g)$ and $\exp _{\xi}^{-1}$ is the geodesic coordinate system. Here, $U_{\mu}$ and $V_{\mu}$ are defined as

$$
U_{\mu}(x)=\mu^{-\frac{N-2}{2}} U\left(\frac{x}{\mu}\right), \quad V_{\mu}(x)=\mu^{-\frac{N-2}{2}} V\left(\frac{x}{\mu}\right),
$$

obtained by scaling $U$ and $V$ in (2.1) and (2.3), respectively. Since $\mu_{g}(M)>0$ implies the coercivity of the conformal laplacian $\Delta_{g}+\alpha_{N} S_{g}$, let $i^{*}: L^{\frac{2 N}{N+2}}(M) \rightarrow H_{g}^{1}(M)$ be the bounded operator defined as follows: the function $u=i^{*}(w)$ is the unique solution in $H_{g}^{1}(M)$ of the equation $\Delta_{g} u+\alpha_{N} S_{g} u=w$ in $M$. Problem (1.4) re-writes as

$$
u=i^{*}\left[u_{+}^{p}-\varepsilon h u\right] \text {, }
$$

and we look for solutions of (2.4) in the form

$$
u_{\varepsilon}(z)=\mathcal{W}_{\mu, \xi}(z)+\phi_{\varepsilon}(z), \quad \mathcal{W}_{\mu, \xi}=\mathcal{U}_{\mu, \xi}+\mu^{2} \mathcal{V}_{\mu, \xi}
$$

where $\xi \in M, \mu>0$ is small and $\phi_{\varepsilon}$ is a small remainder term.

First of all, we introduce the error term

$$
\mathcal{R}_{\mu, \xi}=\mathcal{W}_{\mu, \xi}-i^{*}\left[\left(\mathcal{W}_{\mu, \xi}\right)_{+}^{p}-\varepsilon h \mathcal{W}_{\mu, \xi}\right]
$$

We want to point out that the choice of the ansatz in (2.5) with the extra term $\mathcal{V}_{\mu, \xi}$ is motivated by the need that the error term has to be small enough. Indeed, the error term is estimated as follows.

Lemma 2.1. Let $N \geq 6$. There exists a positive constant $C_{0}>0$ such that for any $\mu$ small and $\xi$ in $M$ there holds

$$
\left\|\mathcal{R}_{\mu, \xi}\right\| \leq C_{0} \begin{cases}\mu^{\frac{N-2}{2}}+\varepsilon \mu^{2}|\ln \mu|^{\frac{2}{3}} & \text { if } N=6 \\ \mu^{\frac{N-2}{2}}+\varepsilon \mu^{2} & \text { if } N=7 \\ \mu^{3}|\ln \mu|^{\frac{5}{8}}+\varepsilon \mu^{2} & \text { if } N=8 \\ \mu^{3}+\varepsilon \mu^{2} & \text { if } N=9 \\ \mu^{2 \frac{N+2}{N-2}}+\varepsilon \mu^{2} & \text { if } N \geq 10 .\end{cases}
$$

Proof. It is enough to estimate the $L^{\frac{2 N}{N+2}}-$ norm of

$$
\Delta_{g} \mathcal{W}_{\mu, \xi}+\left(\alpha_{N} S_{g}+\varepsilon h\right) \mathcal{W}_{\mu, \xi}-\left(\mathcal{W}_{\mu, \xi}\right)_{+}^{p}
$$

Since $\mathcal{U}_{\mu, \xi} \circ \exp _{\xi}$ is radially symmetric in $B_{0}\left(r_{0}\right)$, we have that

$$
\Delta_{g} \mathcal{U}_{\mu, \xi}\left(\exp _{\xi} x\right)=-\Delta\left(\mathcal{U}_{\mu, \xi} \circ \exp _{\xi}\right)(x)-\frac{1}{2} \partial_{r}(\ln |g|) \partial_{r}\left(\mathcal{U}_{\mu, \xi} \circ \exp _{\xi}\right)(x),
$$


where $|g|:=\operatorname{det} g$. In geodesic coordinates, we have the Taylor expansion

$$
|g|=1-\frac{1}{3} \sum_{i, j=1}^{N} R_{i j}(\xi) x^{i} x^{j}+\mathrm{O}\left(|x|^{3}\right)
$$

(see for example Lee-Parker [22]), yielding to

$$
\begin{aligned}
\Delta_{g} \mathcal{U}_{\mu, \xi}\left(\exp _{\xi} x\right)= & -\chi(|x|) \Delta U_{\mu}(x)+\frac{\chi(|x|)}{3} \sum_{i, j=1}^{N} \frac{R_{i j}(\xi) x^{i} x^{j}}{|x|} \partial_{r} U_{\mu}(x) \\
& +\mathrm{O}\left(\mu^{\frac{N-2}{2}}+|x|^{2}\left|\nabla U_{\mu}\right|\right) \\
= & \mathcal{U}_{\mu, \xi}^{p}\left(\exp _{\xi} x\right)+\frac{\chi(|x|)}{3} \sum_{i, j=1}^{N} \frac{R_{i j}(\xi) x^{i} x^{j}}{|x|} \partial_{r} U_{\mu}(x)+\mathrm{O}\left(\mu^{\frac{N-2}{2}}+|x|^{2}\left|\nabla U_{\mu}\right|\right)
\end{aligned}
$$

in view of $-\Delta U_{\mu}=U_{\mu}^{p}$. Similarly, we have that

$$
\Delta_{g} \mathcal{V}_{\mu, \xi}\left(\exp _{\xi} x\right)=-\chi(|x|) \Delta V_{\mu}(x)+\mathrm{O}\left(\mu^{\frac{N-6}{2}}+|x|\left|\nabla V_{\mu}\right|\right)
$$

Since by (2.2) we have that

$$
\Delta\left(\mu^{2} V_{\mu}\right)+p U_{\mu}^{p-1}\left(\mu^{2} V_{\mu}\right)=\frac{1}{3} \sum_{i, j=1}^{N} R_{i j}(\xi) \frac{x^{i} x^{j}}{|x|} \partial_{r} U_{\mu}+\alpha_{N} S_{g}(\xi) U_{\mu},
$$

by (2.9) $-(2.10)$ we get that

$$
\left\|\Delta_{g} \mathcal{W}_{\mu, \xi}+\alpha_{N} S_{g} \mathcal{W}_{\mu, \xi}-\mathcal{U}_{\mu, \xi}^{p}-p \mu^{2} \mathcal{U}_{\mu, \xi}^{p-1} \mathcal{V}_{\mu, \xi}\right\|_{L^{\frac{2 N}{N+2}}(M)}= \begin{cases}\mathrm{O}\left(\mu^{\frac{N-2}{2}}\right) & \text { if } N=6,7 \\ \mathrm{O}\left(\mu^{3}|\ln \mu|^{\frac{5}{8}}\right) & \text { if } N=8 \\ \mathrm{O}\left(\mu^{3}\right) & \text { if } N \geq 9\end{cases}
$$

Since

$$
\left\|h \mathcal{W}_{\mu, \xi}\right\|_{L^{\frac{2 N}{N+2}}(M)}= \begin{cases}\mathrm{O}\left(\mu^{2}|\ln \mu|^{\frac{2}{3}}\right) & \text { if } N=6 \\ \mathrm{O}\left(\mu^{2}\right) & \text { if } N \geq 7\end{cases}
$$

and

$$
\left\|\left(\mathcal{W}_{\mu, \xi}\right)_{+}^{p}-\mathcal{U}_{\mu, \xi}^{p}-p \mathcal{U}_{\mu, \xi}^{p-1}\left(\mu^{2} \mathcal{V}_{\mu, \xi}\right)\right\|_{L^{\frac{2 N}{N+2}}(M)}= \begin{cases}\mathrm{O}\left(\mu^{4}|\ln \mu|^{\frac{2}{3}}\right. & \text { if } N=6 \\ \mathrm{O}\left(\mu^{2 \frac{N+2}{N-2}}\right) & \text { if } N \geq 7\end{cases}
$$

in view of $\left|(a+b)_{+}^{p}-a^{p}-p a^{p-1} b\right|=\mathrm{O}\left(|b|^{p}\right)$ for all $a>0$ and $b \in \mathbb{R}$, by (2.11) we deduce the validity of (2.7).

\section{THE REDUCED ENERGY}

Introduce the Euler-Lagrange functional $J_{\varepsilon}: \mathrm{H}_{g}^{1}(M) \rightarrow \mathbb{R}$ corresponding to equation (1.4):

$$
J_{\varepsilon}(u):=\frac{1}{2} \int_{M}|\nabla u|_{g}^{2} d v_{g}+\frac{1}{2} \int_{M}\left(\alpha_{N} S_{g}+\varepsilon h\right) u^{2} d v_{g}-\frac{1}{p+1} \int_{M} u_{+}^{p+1} d v_{g} .
$$

The aim is to find an asymptotic expansion of $J_{\varepsilon}\left(\mathcal{W}_{\mu, \xi}\right)$. We have that: 
Proposition 3.1. The following expansions do hold as $\epsilon, \mu \rightarrow 0$ :

$$
J_{\varepsilon}\left(\mathcal{W}_{\mu, \xi}\right)=\frac{K_{6}^{-6}}{6}+\frac{4}{5} \omega_{5}\left|\operatorname{Weyl}_{g}(\xi)\right|_{g}^{2} \mu^{4} \ln \mu+\frac{5}{24} K_{6}^{-6} h(\xi) \varepsilon \mu^{2}+\mathrm{o}\left(\mu^{4} \ln \mu+\varepsilon \mu^{2}\right)
$$

when $N=6$, and

$$
\begin{array}{r}
J_{\varepsilon}\left(\mathcal{W}_{\mu, \xi}\right)=\frac{K_{N}^{-N}}{N}-\frac{K_{N}^{-N}}{24 N(N-4)(N-6)}\left|\operatorname{Weyl}_{g}(\xi)\right|_{g}^{2} \mu^{4}+\frac{2(N-1) K_{N}^{-N} h(\xi)}{N(N-2)(N-4)} \varepsilon \mu^{2} \\
+o\left(\mu^{4}+\varepsilon \mu^{2}\right)
\end{array}
$$

when $N \geq 7$, uniformly with respect to $\xi \in M$, where $K_{N}$ is the best constant for the embedding of $D^{1,2}\left(\mathbb{R}^{N}\right)$ into $L^{2^{*}}\left(\mathbb{R}^{N}\right)$.

Proof. First, we have that

$$
\begin{aligned}
& J_{\varepsilon}\left(\mathcal{U}_{\mu, \xi}+\mu^{2} \mathcal{V}_{\mu, \xi}\right)-J_{\varepsilon}\left(\mathcal{U}_{\mu, \xi}\right)=\mu^{2} \int_{M}\left[\left\langle\nabla \mathcal{U}_{\mu, \xi}, \nabla \mathcal{V}_{\mu, \xi}\right\rangle_{g}+\left(\alpha_{N} S_{g}+\varepsilon h\right) \mathcal{U}_{\mu, \xi} \mathcal{V}_{\mu, \xi}-\mathcal{U}_{\mu, \xi}^{p} \mathcal{V}_{\mu, \xi}\right] \\
& \quad+\frac{1}{2} \mu^{4} \int_{M}\left[\left|\nabla \mathcal{V}_{\mu, \xi}\right|_{g}^{2}-p \mathcal{U}_{\mu, \xi}^{p-1} \mathcal{V}_{\mu, \xi}^{2}\right] d v_{g}+\frac{1}{2} \mu^{4} \int_{M}\left(\alpha_{N} S_{g}+\varepsilon h\right) \mathcal{V}_{\mu, \xi}^{2} d v_{g} \\
& \quad-\frac{1}{p+1} \int_{M}\left[\left(\mathcal{U}_{\mu, \xi}+\mu^{2} \mathcal{V}_{\mu, \xi}\right)_{+}^{p+1}-\mathcal{U}_{\mu, \xi}^{p+1}-(p+1) \mathcal{U}_{\mu, \xi}^{p} \mu^{2} \mathcal{V}_{\mu, \xi}-\frac{1}{2} p(p+1) \mathcal{U}_{\mu, \xi}^{p-1} \mu^{4} \mathcal{V}_{\mu, \xi}^{2}\right] d v_{g} \\
& =\mu^{2} \int_{M}\left[\Delta_{g} \mathcal{U}_{\mu, \xi}+\alpha_{N} S_{g} \mathcal{U}_{\mu, \xi}-\mathcal{U}_{\mu, \xi}^{p}\right] \mathcal{V}_{\mu, \xi} d v_{g}+\frac{1}{2} \mu^{4} \int_{M}\left[\Delta_{g} \mathcal{V}_{\mu, \xi}-p \mathcal{U}_{\mu, \xi}^{p-1} \mathcal{V}_{\mu, \xi}\right] \mathcal{V}_{\mu, \xi} d v_{g} \\
& + \begin{cases}0\left(\mu^{4} \ln \mu\right) & \text { if } N=6 \\
0\left(\mu^{4}\right) & \text { if } N \geq 7\end{cases}
\end{aligned}
$$

as $\mu \rightarrow 0$, in view of

$$
\begin{gathered}
\int_{M}\left|\left(\mathcal{U}_{\mu, \xi}+\mu^{2} \mathcal{V}_{\mu, \xi}\right)_{+}^{p+1}-\mathcal{U}_{\mu, \xi}^{p+1}-(p+1) \mathcal{U}_{\mu, \xi}^{p} \mu^{2} \mathcal{V}_{\mu, \xi}-\frac{1}{2} p(p+1) \mathcal{U}_{\mu, \xi}^{p-1} \mu^{4} \mathcal{V}_{\mu, \xi}^{2}\right| d v_{g} \\
=\mathrm{O}\left(\mu^{\frac{4 N}{N-2}} \int_{M}\left|\mathcal{V}_{\mu, \xi}\right|^{\frac{2 N}{N-2}} d v_{g}\right)=\mathrm{o}\left(\mu^{4}\right)
\end{gathered}
$$

and $\int_{M} \mathcal{V}_{\mu, \xi}^{2} d v_{g}=\left\{\begin{array}{ll}\mathrm{O}(1) & \text { if } N=6 \\ \mathrm{o}(1) & \text { if } N \geq 7\end{array}\right.$ as $\mu \rightarrow 0$. Now, observe that there holds

$$
\begin{aligned}
& \mu^{2} \int_{M}\left[\Delta_{g} \mathcal{U}_{\mu, \xi}+\alpha_{N} S_{g} \mathcal{U}_{\mu, \xi}-\mathcal{U}_{\mu, \xi}^{p}\right] \mathcal{V}_{\mu, \xi} d v_{g}+\mu^{4} \int_{M}\left[\Delta_{g} \mathcal{V}_{\mu, \xi}-p \mathcal{U}_{\mu, \xi}^{p-1} \mathcal{V}_{\mu, \xi}\right] \mathcal{V}_{\mu, \xi} d v_{g} \\
& =\mu^{2} \int_{M}\left[\Delta_{g} \mathcal{W}_{\mu, \xi}+\alpha_{N} S_{g} \mathcal{W}_{\mu, \xi}-\mathcal{U}_{\mu, \xi}^{p}-p \mu^{2} \mathcal{U}_{\mu, \xi}^{p-1} \mathcal{V}_{\mu, \xi}\right] \mathcal{V}_{\mu, \xi} d v_{g}+\mathrm{O}\left(\mu^{4} \int_{M} \mathcal{V}_{\mu, \xi}^{2} d v_{g}\right) \\
& = \begin{cases}\mathrm{o}\left(\mu^{4} \ln \mu\right) & \text { if } N=6 \\
\mathrm{o}\left(\mu^{4}\right) & \text { if } N \geq 7\end{cases}
\end{aligned}
$$

as $\mu \rightarrow 0$, in view of (2.11). By (2.8) and

$$
\begin{aligned}
\Delta_{g} \mathcal{V}_{\mu, \xi}\left(\exp _{\xi} x\right)= & -\Delta\left(\mathcal{V}_{\mu, \xi} \circ \exp _{\xi}\right)(x) \\
& +\mathrm{O}\left(|x|\left|\nabla\left(\mathcal{V}_{\mu, \xi} \circ \exp _{\xi}\right)(x)\right|+|x|^{2}\left|\nabla^{2}\left(\mathcal{V}_{\mu, \xi} \circ \exp _{\xi}\right)(x)\right|\right)
\end{aligned}
$$

we deduce that

$$
\int_{M}\left[\Delta_{g} \mathcal{V}_{\mu, \xi}-p \mathcal{U}_{\mu, \xi}^{p-1} \mathcal{V}_{\mu, \xi}\right] \mathcal{V}_{\mu, \xi} d v_{g}=-\int_{B_{0}\left(\frac{r_{0}}{2 \mu}\right)}\left(\Delta V+p U^{p-1} V\right) V d y+ \begin{cases}\mathrm{O}(1) & \text { if } N=6 \\ \mathrm{o}(1) & \text { if } N \geq 7\end{cases}
$$



as $\mu \rightarrow 0$. By (3.3) and (3.4), we get that

$$
J_{\varepsilon}\left(\mathcal{U}_{\mu, \xi}+\mu^{2} \mathcal{V}_{\mu, \xi}\right)=J_{\varepsilon}\left(\mathcal{U}_{\mu, \xi}\right)+\frac{1}{2} \mu^{4} \int_{B_{0}\left(\frac{r_{0}}{2 \mu}\right)}\left(\Delta V+p U^{p-1} V\right) V d y+ \begin{cases}\mathrm{o}\left(\mu^{4} \ln \mu\right) & \text { if } N=6 \\ \mathrm{o}\left(\mu^{4}\right) & \text { if } N \geq 7\end{cases}
$$

as $\mu \rightarrow 0$. By (2.2) $-(2.3)$ and easy symmetry properties we deduce that

$$
\begin{aligned}
\int_{B_{0}\left(\frac{r_{0}}{2 \mu}\right)}\left(\Delta V+p U^{p-1} V\right) V d y & \\
= & -\frac{[N(N-2)]^{\frac{N-2}{2}}(N-2)}{36} \int_{B_{0}\left(\frac{r_{0}}{2 \mu}\right)}\left(\sum_{i, j=1}^{N} R_{i j}(\xi) y^{i} y^{j}\right)^{2} \frac{|y|^{2}+3}{\left(1+|y|^{2}\right)^{N}} d y \\
+ & \frac{[N(N-2)]^{\frac{N-2}{2}} \alpha_{N}}{72 N(N-1)} S_{g}^{2}(\xi) \int_{B_{0}\left(\frac{r_{0}}{2 \mu}\right)} \frac{(7 N-10)|y|^{6}+3(7 N-8)|y|^{4}+3(7 N-10)|y|^{2}-9 N}{\left(1+|y|^{2}\right)^{N}} d y \\
= & -\frac{[N(N-2)]^{\frac{N-2}{2}}(N-2)}{36} \int_{B_{0}\left(\frac{r_{0}}{2 \mu}\right)} \sum_{i, j, k, s=1}^{N} E_{i j}(\xi) E_{k s}(\xi) y^{i} y^{j} y^{k} y^{s} \frac{|y|^{2}+3}{\left(1+|y|^{2}\right)^{N}} d y \\
& -\omega_{N-1} \frac{[N(N-2)]^{\frac{N-2}{2}}(N-2)}{576 N^{2}(N-1)^{2}} S_{g}^{2}(\xi)\left[(N-2)(N-4) I_{N}^{\frac{N+4}{2}}+3\left(N^{2}-8 N+8\right) I_{N}^{\frac{N+2}{2}}\right. \\
& \left.-3 N(7 N-10) I_{N}^{\frac{N}{2}}+9 N^{2} I_{N}^{\frac{N-2}{2}}\right]+\mathrm{o}(1)
\end{aligned}
$$

as $\mu \rightarrow 0$, where the $E_{i j}$ 's are the components of the traceless part $\mathrm{E}_{g}=\mathrm{Ric}_{g}-\frac{S_{g}}{N} g$ of the Ricci curvature $\operatorname{Ric}_{g}$ of $(M, g)$ in geodesic coordinates and

$$
I_{p}^{q}= \begin{cases}\int_{0}^{+\infty} \frac{r^{q}}{(1+r)^{p}} d r & \text { if } p-q>1 \\ \int_{0}^{\frac{r_{0}^{2}}{4 \mu^{2}}} \frac{r^{q}}{(1+r)^{p}} d r & \text { if } p-q \leq 1\end{cases}
$$

Since integration by parts yields to

$$
I_{p+1}^{q}=\frac{p-q-1}{p} I_{p}^{q} \quad \text { and } \quad I_{p+1}^{q+1}=\frac{q+1}{p-q-1} I_{p+1}^{q}
$$

as soon as $p-q>1$, we have that

$$
I_{N}^{\frac{N}{2}}=\frac{N}{N-2} I_{N}^{\frac{N-2}{2}}=\frac{N-4}{N+2} I_{N}^{\frac{N+2}{2}} \quad \text { and } \quad I_{N}^{\frac{N+4}{2}}= \begin{cases}-2 \ln \mu+\mathrm{O}(1) & \text { if } N=6 \\ \frac{(N+2)(N+4)}{(N-4)(N-6)} I_{N}^{\frac{N}{2}} & \text { if } N \geq 7\end{cases}
$$

as $\mu \rightarrow 0$, and it can be easily checked that

$$
I_{N}^{\frac{N}{2}}=\frac{N \omega_{N}}{2^{N-1}(N-2) \omega_{N-1}}=\frac{2 K_{N}^{-N}}{[N(N-2)]^{\frac{N-2}{2}}(N-2)^{2} \omega_{N-1}}
$$

(see Aubin [1]). Since for all $i \neq j$ there holds

$$
\int_{S^{N-1}}\left(y^{i}\right)^{4} d v_{g_{0}}=3 \int_{S^{N-1}}\left(y^{i}\right)^{2}\left(y^{j}\right)^{2} d v_{g_{0}}=\frac{3}{N(N+2)} \int_{S^{N-1}}|y|^{4} d v_{g_{0}},
$$


by (3.6) and (3.8)-(3.9) we deduce that

$$
\int_{B_{0}\left(\frac{r_{0}}{2 \mu}\right)}\left(\Delta V+p U^{p-1} V\right) V d y=\frac{8}{3} \omega_{5}\left|E_{g}(\xi)\right|_{g}^{2} \ln \mu+\frac{16}{225} \omega_{5} S_{g}^{2}(\xi) \ln \mu+\mathrm{O}(1)
$$

if $N=6$, and

$$
\begin{aligned}
\int_{B_{0}\left(\frac{r_{0}}{2 \mu}\right)}\left(\Delta V+p U^{p-1} V\right) V d y=- & \frac{2 N-7}{9 N(N-2)(N-4)(N-6)} K_{N}^{-N}\left|E_{g}(\xi)\right|_{g}^{2} \\
& +\frac{(N-2)(N-7)}{36 N^{2}(N-1)(N-4)(N-6)} K_{N}^{-N} S_{g}^{2}(\xi)+\mathrm{o}(1)
\end{aligned}
$$

if $N \geq 7$. Inserting (3.10)-(3.11) into (3.5), by Lemma 3.2 below we deduce the validity of (3.1)-(3.2).

We are left with proving the following:

Lemma 3.2. The following expansions do hold as $\epsilon, \mu \rightarrow 0$ :

$$
\begin{aligned}
J_{\varepsilon}\left(U_{\mu, \xi}\right)= & \frac{K_{6}^{-6}}{6}+\left[\frac{4}{5}\left|\operatorname{Weyl}_{g}(\xi)\right|_{g}^{2}-\frac{4}{3}\left|E_{g}(\xi)\right|_{g}^{2}-\frac{8}{225} S_{g}^{2}(\xi)\right] \omega_{5} \mu^{4} \ln \mu+\frac{5}{24} K_{6}^{-6} h(\xi) \varepsilon \mu^{2} \\
& +\mathrm{o}\left(\mu^{4} \ln \mu+\varepsilon \mu^{2}\right)
\end{aligned}
$$

when $N=6$, and

$$
\begin{aligned}
J_{\varepsilon}\left(U_{\mu, \xi}\right)= & \frac{K_{N}^{-N}}{N}+\left[-\frac{K_{N}^{-N}}{24 N(N-4)(N-6)}\left|\operatorname{Weyl}_{g}(\xi)\right|_{g}^{2}\right. \\
& \left.+\frac{(2 N-7) K_{N}^{-N}}{18 N(N-2)(N-4)(N-6)}\left|\mathrm{E}_{g}(\xi)\right|_{g}^{2}-\frac{(N-2)(N-7) K_{N}^{-N}}{72 N^{2}(N-1)(N-4)(N-6)} S_{g}(\xi)^{2}\right] \mu^{4} \\
& +\frac{2(N-1) K_{N}^{-N}}{N(N-2)(N-4)} h(\xi) \varepsilon \mu^{2}+\mathrm{o}\left(\mu^{4}+\varepsilon \mu^{2}\right)
\end{aligned}
$$

when $N \geq 7$, uniformly with respect to $\xi \in M$.

Proof. There hold

$$
\begin{aligned}
& \frac{1}{\omega_{N-1} r^{N-1}} \int_{\partial B_{\xi}(r)} h d \sigma_{g}=h(\xi)+\mathrm{O}(r), \\
& \frac{1}{\omega_{N-1} r^{N-1}} \int_{\partial B_{\xi}(r)} S_{g} d \sigma_{g}=S_{g}(\xi)-\frac{1}{2 N} \Lambda_{g}(\xi) r^{2}+\mathrm{O}\left(r^{4}\right), \\
& \frac{1}{\omega_{N-1} r^{N-1}} \int_{\partial B_{\xi}(r)} d \sigma_{g}=1-\frac{1}{6 N} S_{g}(\xi) r^{2}+A_{g}(\xi) r^{4}+\mathrm{O}\left(r^{5}\right),
\end{aligned}
$$

as $r \rightarrow 0$, uniformly with respect to $\xi$, where $d \sigma_{g}$ is the volume element of $\partial B_{\xi}(r), \omega_{N-1}$ is the volume of the unit $(N-1)$-sphere, and where (see (3.17)-(3.18) $)$

$$
\Lambda_{g}(\xi)=\Delta_{g} S_{g}(\xi)+\frac{1}{3} S_{g}(\xi)^{2}
$$

and

$$
A_{g}(\xi)=\frac{18 \Delta_{g} S_{g}(\xi)+8\left|\operatorname{Ric}_{g}(\xi)\right|_{g}^{2}-3\left|\mathrm{Rm}_{g}(\xi)\right|_{g}^{2}+5 S_{g}(\xi)^{2}}{360 N(N+2)}
$$


The orthogonal decomposition of Riemann curvature is given by

$$
\left|\operatorname{Rm}_{g}(\xi)\right|_{g}^{2}=\left|\operatorname{Weyl}_{g}(\xi)\right|_{g}^{2}+\frac{4}{N-2}\left|\mathrm{E}_{g}(\xi)\right|_{g}^{2}+\frac{2}{N(N-1)} S_{g}(\xi)^{2}
$$

where Weyl $g$ is the Weyl curvature of $g$ and $\mathrm{E}_{g}=\operatorname{Ric}_{g}-\frac{S_{g}}{N} g$ is the traceless part of the Ricci curvature of $g$. Moreover, we get

$$
\left|\operatorname{Ric}_{g}(\xi)\right|_{g}^{2}=\left|\mathrm{E}_{g}(\xi)\right|_{g}^{2}+\frac{1}{N} S_{g}(\xi)^{2}
$$

By (3.8) and (3.14), we compute

$$
\begin{aligned}
\int_{M} \mid & \left.\nabla U_{\mu, \xi}\right|_{g} ^{2} d v_{g}=[N(N-2)]^{\frac{N-2}{2}}(N-2)^{2} \int_{0}^{\frac{r_{0}}{2}} \frac{\mu^{N-2} r^{2}}{\left(\mu^{2}+r^{2}\right)^{N}} \int_{\partial B_{\xi}(r)} d \sigma_{g} d r+\mathrm{O}\left(\mu^{N-2}\right) \\
= & {[N(N-2)]^{\frac{N-2}{2}}(N-2)^{2} \omega_{N-1} } \\
& \times \int_{0}^{\frac{r_{0}}{2 \mu}} \frac{r^{N+1}}{\left(1+r^{2}\right)^{N}}\left(1-\frac{1}{6 N} S_{g}(\xi) \mu^{2} r^{2}+A_{g}(\xi) \mu^{4} r^{4}+\mathrm{O}\left(\mu^{5} r^{5}\right)\right) d r+\mathrm{O}\left(\mu^{N-2}\right) \\
= & \frac{[N(N-2)]^{\frac{N-2}{2}}(N-2)^{2}}{2} \omega_{N-1} \\
& \times\left(I_{N}^{\frac{N}{2}}-\frac{1}{6 N} I_{N}^{\frac{N+2}{2}} S_{g}(\xi) \mu^{2}+I_{N}^{\frac{N+4}{2}} A_{g}(\xi) \mu^{4}+\mathrm{O}\left(I_{N}^{\frac{N+5}{2}} \mu^{5}+\mu^{N-2}\right)\right) \\
= & \left\{\begin{array}{l}
K_{N}^{-N}\left(1-\frac{N+2}{6 N(N-4)} S_{g}(\xi) \mu^{2}\right)-9216 \omega_{5} A_{g}(\xi) \mu^{4} \ln \mu+\mathrm{O}\left(\mu^{4}\right) \\
K_{N}^{-N}\left(1-\frac{N+2}{6 N(N-4)} S_{g}(\xi) \mu^{2}+\frac{(N+2)(N+4)}{(N-4)(N-6)} A_{g}(\xi) \mu^{4}\right)+\mathrm{O}\left(\mu^{5}\right) \quad \text { if } N \geq 7
\end{array}\right.
\end{aligned}
$$

in view of (3.9) $)$. Since by (3.7) there hold

$$
I_{N-2}^{\frac{N-2}{2}}=\frac{4(N-1)(N-2)}{N(N-4)} I_{N}^{\frac{N}{2}} \quad \text { and } \quad I_{N-2}^{\frac{N}{2}}= \begin{cases}-2 \ln \mu+\mathrm{O}(1) & \text { if } N=6 \\ \frac{4(N-1)(N-2)}{(N-4)(N-6)} I_{N}^{\frac{N}{2}} & \text { if } N \geq 7\end{cases}
$$

as $\mu \rightarrow 0$, by (3.13) we compute

$$
\begin{aligned}
& \int_{M} S_{g} U_{\mu, \xi}^{2} d v_{g}=[N(N-2)]^{\frac{N-2}{2}} \int_{0}^{\frac{r_{0}}{2}} \frac{\mu^{N-2}}{\left(\mu^{2}+r^{2}\right)^{N-2}} \int_{\partial B_{\xi}(r)} S_{g} d \sigma_{g} d r+\mathrm{O}\left(\mu^{N-2}\right) \\
& =[N(N-2)]^{\frac{N-2}{2}} \omega_{N-1} \mu^{2} \int_{0}^{\frac{r_{0}}{2 \mu}} \frac{r^{N-1}}{\left(1+r^{2}\right)^{N-2}}\left(S_{g}(\xi)-\frac{1}{2 N} \Lambda_{g}(\xi) \mu^{2} r^{2}+\mathrm{O}\left(\mu^{4} r^{4}\right)\right) d r \\
& +\mathrm{O}\left(\mu^{N-2}\right) \\
& =\frac{[N(N-2)]^{\frac{N-2}{2}}}{2} \omega_{N-1} \mu^{2}\left(I_{N-2}^{\frac{N-2}{2}} S_{g}(\xi)-\frac{1}{2 N} I_{N-2}^{\frac{N}{2}} \Lambda_{g}(\xi) \mu^{2}+\mathrm{O}\left(\mu^{4} I_{N-2}^{\frac{N+2}{2}}+\mu^{N-2}\right)\right) \\
& = \begin{cases}\frac{5 K_{6}^{-6}}{12} \mu^{2} S_{g}(\xi)+48 \omega_{5} \Lambda_{g}(\xi) \mu^{4} \ln \mu+\mathrm{O}\left(\mu^{4}\right) & \text { if } N=6 \\
\frac{4(N-1) K_{N}^{-N}}{N(N-2)(N-4)} \mu^{2}\left(S_{g}(\xi)-\frac{1}{2(N-6)} \Lambda_{g}(\xi) \mu^{2}\right)+\mathrm{O}\left(\mu^{5}\right) & \text { if } N \geq 7\end{cases}
\end{aligned}
$$


in view of (3.9). Similarly, by (3.12), we have that

$$
\varepsilon \int_{M} h U_{\mu, \xi}^{2} d v_{g}=\frac{4(N-1) K_{N}^{-N}}{N(N-2)(N-4)} h(\xi) \varepsilon \mu^{2}+\mathrm{o}\left(\varepsilon \mu^{2}\right)
$$

By (3.8) and (3.14), we compute

$$
\begin{aligned}
& \int_{M} U_{\mu, \xi}^{2^{*}} d v_{g}=[N(N-2)]^{\frac{N}{2}} \int_{0}^{\frac{r_{0}}{2}} \frac{\mu^{N}}{\left(\mu^{2}+r^{2}\right)^{N}} \int_{\partial B_{\xi}(r)} d \sigma_{g} d r+\mathrm{O}\left(\mu^{N}\right) \\
& =[N(N-2)]^{\frac{N}{2}} \omega_{N-1} \int_{0}^{\frac{r_{0}}{2 \mu}} \frac{r^{N-1}}{\left(1+r^{2}\right)^{N}}\left(1-\frac{1}{6 N} S_{g}(\xi) \mu^{2} r^{2}+A_{g}(\xi) \mu^{4} r^{4}\right) d r+\mathrm{O}\left(\mu^{5}\right) \\
& =\frac{[N(N-2)]^{\frac{N}{2}}}{2} \omega_{N-1}\left(I_{N}^{\frac{N-2}{2}}-\frac{1}{6 N} I_{N}^{\frac{N}{2}} S_{g}(\xi) \mu^{2}+I_{N}^{\frac{N+2}{2}} A_{g}(\xi) \mu^{4}\right)+\mathrm{O}\left(\mu^{5}\right) \\
& =K_{N}^{-N}\left(1-\frac{1}{6(N-2)} S_{g}(\xi) \mu^{2}+\frac{N(N+2)}{(N-2)(N-4)} A_{g}(\xi) \mu^{4}\right)+\mathrm{O}\left(\mu^{5}\right)
\end{aligned}
$$

in view of (3.9). Finally, the claimed expansions follow by (3.19), (3.20), (3.21) and (3.22) in view of (3.15)-(3.18) .

\section{The Lyapunov-Schmidt Reduction ARgument}

Since equation (1.4) can be re-written as (2.4), the function $u=\mathcal{W}_{\mu, \xi}+\phi$ does solve (1.4) as soon as

$$
\hat{L}_{\mu, \xi}(\phi)=-\mathcal{R}_{\mu, \xi}-N_{\mu, \xi}(\phi)
$$

where $\mathcal{R}_{\mu, \xi}$ is given in (2.6),

$$
N_{\mu, \xi}(\phi)=-i^{*}\left[\left(\mathcal{W}_{\mu, \xi}+\phi\right)_{+}^{p}-\left(\mathcal{W}_{\mu, \xi}\right)_{+}^{p}-p\left(\mathcal{W}_{\mu, \xi}\right)_{+}^{p-1} \phi\right]
$$

is the nonlinear term (quadratic in $\phi$ ) and

$$
\begin{aligned}
\hat{L}_{\mu, \xi}: H_{g}^{1}(M) & \rightarrow H_{g}^{1}(M) \\
\phi & \mapsto \phi-i^{*}\left[p\left(\mathcal{W}_{\mu, \xi}\right)_{+}^{p-1} \phi-\varepsilon h \phi\right]
\end{aligned}
$$

is the linearized operator of $(2.4)$ at $\mathcal{W}_{\mu, \xi}$.

Since $\mathcal{W}_{\mu, \xi}$ is a small perturbation of $\mathcal{U}_{\mu, \xi}$, as $\varepsilon, \mu \rightarrow 0$ the operator $\hat{L}_{\mu, \xi}$ in balls with radii of order $\mu$ looks pretty much as a scaling of the limiting operator $L_{\infty}: \Phi \rightarrow \Phi+(\Delta)^{-1}\left[p U^{p-1} \Phi\right]$, where $U$ is given in (2.1). It is well known (see Bianchi-Egnell [4]) that

$$
\text { ker } L_{\infty}=\operatorname{Span}\left\{\Phi^{0}, \Phi^{1}, \ldots, \Phi^{N}\right\} \text {, }
$$

where

$$
\Phi^{0}(y)=\frac{1-|y|^{2}}{\left(1+|y|^{2}\right)^{\frac{N}{2}}}, \quad \Phi^{i}(y)=\frac{y^{i}}{\left(1+|y|^{2}\right)^{\frac{N}{2}}} \quad \forall i=1, \ldots, N .
$$

Since there is no hope for the full invertibility of $\hat{L}_{\mu, \xi}$ in $H_{g}^{1}(M)$, let us introduce the "asymptotic kernel" $K_{\mu, \xi}$ and its "orthogonal space" $K_{\mu, \xi}^{\perp}$ as

$$
K_{\mu, \xi}=\operatorname{Span}\left\{Z_{\mu, \xi}^{0}, \ldots, Z_{\mu, \xi}^{N}\right\}
$$

and

$$
K_{\mu, \xi}^{\perp}=\left\{\phi \in H_{g}^{1}(M): \int_{M} \mathcal{U}_{\mu, \xi}^{p-1} Z_{\mu, \xi}^{i} \phi d \mu_{g}=0 \quad \forall i=0, \ldots, N\right\},
$$


where

$$
Z_{\mu, \xi}^{i}(z)=\chi\left(d_{g}(z, \xi)\right) \mu^{\frac{2-N}{2}} \Phi^{i}\left(\frac{\exp _{\xi}^{-1}(z)}{\mu}\right)
$$

for $i=0, \ldots, N$, with $\Phi^{i}$ given by (4.2). Letting $\Pi_{\mu, \xi}$ and $\Pi_{\mu, \xi}^{\perp}$ be the projectors of $H_{g}^{1}(M)$ onto the respective subspaces, equation (4.1) is equivalent to solving

$$
\begin{aligned}
& L_{\mu, \xi}(\phi)=-\Pi_{\mu_{\xi}}^{\perp}\left(\mathcal{R}_{\mu, \xi}+N_{\mu, \xi}(\phi)\right), \\
& \Pi_{\mu_{\xi}}\left(\hat{L}_{\mu, \xi}(\phi)\right)=-\Pi_{\mu_{\xi}}\left(\mathcal{R}_{\mu, \xi}+N_{\mu, \xi}(\phi)\right)
\end{aligned}
$$

for some $\phi \in K_{\mu, \xi}^{\perp}$, where $L_{\mu, \xi}=\Pi_{\mu, \xi}^{\perp} \circ \hat{L}_{\mu, \xi}: K_{\mu, \xi}^{\perp} \rightarrow K_{\mu, \xi}^{\perp}$. First we can solve equation (4.3), a rather standard result in this context (see for example Musso-Pistoia [30]):

Lemma 4.1. There exists a positive constant $C_{0}$ such that, for any $\varepsilon, \mu$ small and any $\xi \in M$, there holds

$$
\left\|L_{\mu, \xi}(\phi)\right\| \geq C_{0}\|\phi\|
$$

for all $\phi \in K_{\mu, \xi}^{\perp}$. As a consequence, (4.3) admits a unique solution $\phi_{\mu, \xi} \in K_{\mu, \xi}^{\perp}$, which is continuously differentiable in $\mu$ and $\xi$, so that

$$
\left\|\phi_{\mu, \xi}\right\|= \begin{cases}\mathrm{o}\left(\mu^{2} \sqrt{|\ln \mu|}+\sqrt{\varepsilon} \mu\right) & \text { if } N=6 \\ \mathrm{o}\left(\mu^{2}+\sqrt{\varepsilon} \mu\right) & \text { if } N \geq 7 .\end{cases}
$$

Let us just stress out that the estimate (4.5) heavily depends on (2.7). The need of improving the ansatz in Section 2 comes out from getting the correct smallness rate of $\phi$ as expressed by (4.5). Finally, we have all the ingredients to prove our main result.

Proof of Theorem 1.2. A first well known fact (see for example Musso-Pistoia [30]) is the equivalence between equation (4.4) and the search of critical points for

$$
\tilde{\mathcal{J}}_{\varepsilon}(\mu, \xi)=J_{\varepsilon}\left(\mathcal{W}_{\mu, \xi}+\phi_{\mu, \xi}\right),
$$

where $\phi_{\mu, \xi}$ is given by Lemma 4.1. We just need to prove that

$$
J_{\varepsilon}\left(\mathcal{W}_{\mu, \xi}+\phi_{\mu, \xi}\right)-J_{\varepsilon}\left(\mathcal{W}_{\mu, \xi}\right)= \begin{cases}\mathrm{o}\left(\mu^{4}|\ln \mu|+\varepsilon \mu^{2}\right) & \text { if } N=6 \\ \mathrm{o}\left(\mu^{4}+\varepsilon \mu^{2}\right) & \text { if } N \geq 7\end{cases}
$$

as $\varepsilon, \mu \rightarrow 0$. Indeed, we have that

$$
\begin{aligned}
J_{\varepsilon}\left(\mathcal{W}_{\mu, \xi}+\phi_{\mu, \xi}\right)-J_{\varepsilon}\left(\mathcal{W}_{\mu, \xi}\right)=\int_{M}\left(\left\langle\nabla \mathcal{R}_{\mu, \xi}, \nabla \phi_{\mu, \xi}\right\rangle_{g}+\alpha_{N} S_{g} \mathcal{R}_{\mu, \xi} \phi_{\mu, \xi}-\left(W_{\mu, \xi}\right)_{+}^{p} \phi_{\mu, \xi}\right) d v_{g} \\
\quad+\frac{1}{2} \int_{M}\left|\nabla \phi_{\mu, \xi}\right|_{g}^{2} d v_{g}+\frac{1}{2} \int_{M}\left(\alpha_{N} S_{g}+\varepsilon h\right) \phi_{\mu, \xi}^{2} d v_{g} \\
\quad-\frac{1}{p+1} \int_{M}\left[\left(\mathcal{W}_{\mu, \xi}+\phi_{\mu, \xi}\right)_{+}^{p+1}-\left(\mathcal{W}_{\mu, \xi}\right)_{+}^{p+1}-(p+1)\left(\mathcal{W}_{\mu, \xi}\right)_{+}^{p} \phi_{\mu, \xi}\right] d v_{g} \\
=\mathrm{O}\left(\left\|\mathcal{R}_{\mu, \xi}\right\|\left\|\phi_{\mu, \xi}\right\|+\left\|\phi_{\mu, \xi}\right\|^{2}+\int_{M}\left(\mathcal{W}_{\mu, \xi}\right)_{+}^{p-1} \phi_{\mu, \xi}^{2} d v_{g}+\int_{M} \phi_{\mu, \xi}^{p+1} d v_{g}\right) \\
=\mathrm{O}\left(\left\|\mathcal{R}_{\mu, \xi}\right\|\left\|\phi_{\mu, \xi}\right\|+\left\|\phi_{\mu, \xi}\right\|^{2}\right)
\end{aligned}
$$

by the Sobolev embedding $H_{g}^{1}(M) \hookrightarrow L^{p+1}(M)$ and the Hölder's inequality. By (2.7) and (4.5) we then deduce the validity of (4.6). Setting

$$
\mu(d)=d \begin{cases}l^{-1}(\varepsilon) & \text { if } N=6 \\ \sqrt{\varepsilon} & \text { if } N \geq 7\end{cases}
$$


where $l:\left(0, e^{-\frac{1}{2}}\right) \rightarrow\left(0, \frac{e^{-1}}{2}\right)$ is defined as $l(\mu)=-\mu^{2} \ln \mu$, by Proposition 3.1 and (4.6) we deduce the following asymptotic estimates:

$$
\mathcal{J}(d, \xi):=\frac{\widetilde{\mathcal{J}}_{\varepsilon}(\mu(d), \xi)-K_{N}^{-N}}{\varepsilon^{2}}\left(\ln l^{-1}(\varepsilon)\right)^{\gamma}=c_{2} d^{2} h(\xi)-c_{3} d^{4} \mid \text { Weyl }\left._{g}(\xi)\right|_{g} ^{2}+\mathrm{o}(1)
$$

as $\varepsilon \rightarrow 0$, uniformly with respect to $\xi \in M$ and to $d$ in compact subsets of $(0, \infty)$, where $c_{2}, c_{3}>0$ are suitable constants, $\gamma=1$ when $N=6$ and $\gamma=0$ when $N \geq 7$. Letting $\mathcal{D} \subset(0, \infty) \times M$ be a $C^{0}$-stable critical set of $\widetilde{E}$ and $U$ be a compact neighborhood of $\mathcal{D}$ in $(0, \infty) \times M$, by the definition of stability it follows that $\mathcal{J}$ has a critical point $\left(d_{\varepsilon}, \xi_{\varepsilon}\right) \in$ $U \subset(0, \infty) \times M$, for $\varepsilon$ small. Up to a subsequence and taking $U$ smaller and smaller, we can assume that $\left(d_{\varepsilon}, \xi_{\varepsilon}\right) \rightarrow\left(t_{0}, \xi_{0}\right)$ as $\varepsilon \rightarrow 0$ with $\xi_{0} \in \pi(\mathcal{D})$. By elliptic regularity theory $u_{\varepsilon}=\mathcal{W}_{\mu\left(d_{\varepsilon}\right), \xi_{\varepsilon}}+\phi_{\mu\left(d_{\varepsilon}\right), \xi_{\varepsilon}}$ is a solution of (1.4). Since $\xi_{\varepsilon} \rightarrow \xi_{0}$ and $\left\|\phi_{\mu\left(d_{\varepsilon}\right), \xi_{\varepsilon}}\right\| \rightarrow 0$ as $\varepsilon \rightarrow 0$, it is easily seen that $u_{\varepsilon}>0$ and $u_{\varepsilon}^{2^{*}} \rightarrow K_{N}^{-N} \delta_{\xi_{0}}$ in the measures sense as $\varepsilon \rightarrow 0$ (see for example Rey [35]), where $\delta_{\xi}$ denotes the Dirac mass measure at $\xi$. From very basic facts concerning the asymptotic analysis of solutions of Yamabe-type equations (see for example Druet-Hebey [12] and Druet-Hebey-Robert[15]), we get that the family $\left(u_{\varepsilon}\right)_{\varepsilon}$ blows up at the point $\xi_{0}$ as $\varepsilon \rightarrow 0$.

\section{REFERENCES}

[1] T. Aubin, Equations différentielles non linéaires et problème de Yamabe concernant la courbure scalaire, J. Math. Pures Appl. (9) 55 (1976), no. 3, 269-296.

[2] A. Bahri, Y.-Y. Li, and O. Rey, On a variational problem with lack of compactness: the topological effect of the critical points at infinity, Calc. Var. Partial Differential Equations 3 (1995), no. 1, 67-93.

[3] M. Ben Ayed, K. El Mehdi, O. Rey, and M. Grossi, A nonexistence result of single peaked solutions to a supercritical nonlinear problem, Commun. Contemp. Math. 5 (2003), no. 2, 179-195.

[4] G. Bianchi and H. Egnell, A note on the Sobolev inequality, J. Funct. Anal. 100 (1991), no. 1, 18-24.

[5] S. Brendle, Blow-up phenomena for the Yamabe equation, J. Amer. Math. Soc. 21 (2008), no. 4, $951-979$.

[6] S. Brendle and F. C. Marques, Blow-up phenomena for the Yamabe equation. II, J. Differential Geom. 81 (2009), no. 2, 225-250.

[7] H. Brézis and L. Nirenberg, Positive solutions of nonlinear elliptic equations involving critical Sobolev exponents, Comm. Pure Appl. Math. 36 (1983), no. 4, 437-477.

[8] W.-Y. Chen, J.-C. Wei, and S.-S. Yan, Infinitely many solutions for the Schrödinger equations in $\mathbb{R}^{n}$ with critical growth, J. Differential Equations.

[9] O. Druet, From one bubble to several bubbles: the low-dimensional case, J. Differential Geom. 63 (2003), no. 3, 399-473.

[10] _ Compactness for Yamabe metrics in low dimensions, Int. Math. Res. Not. 23 (2004), $1143-1191$.

[11] O. Druet and E. Hebey, Blow-up examples for second order elliptic PDEs of critical Sobolev growth, Trans. Amer. Math. Soc. 357 (2005), no. 5, 1915-1929 (electronic).

[12] _ Elliptic equations of Yamabe type, Int. Math. Res. Surv. 1 (2005), 1-113.

[13] Stability for strongly coupled critical elliptic systems in a fully inhomogeneous medium, Anal. PDE 2 (2009), no. 3, 305-359.

[14] _ Stability and instability for Einstein-scalar field Lichnerowicz equations on compact Riemannian manifolds, Math. Z. 263 (2009), no. 1, 33-67.

[15] O. Druet, E. Hebey, and F. Robert, Blow-up theory for elliptic PDEs in Riemannian geometry, Mathematical Notes, vol. 45, Princeton University Press, Princeton, NJ, 2004.

[16] O. Druet, E. Hebey, and J. Vétois, Bounded stability for strongly coupled critical elliptic systems below the geometric threshold of the conformal Laplacian, J. Funct. Anal. 258 (2010), no. 3, 999-1059.

[17] P. Esposito, A. Pistoia, and J. Vétois, The effect of linear perturbations on the Yamabe problem (2012). Preprint.

[18] Z.-C. Han, Asymptotic approach to singular solutions for nonlinear elliptic equations involving critical Sobolev exponent, Ann. Inst. H. Poincaré Anal. Non Linéaire 8 (1991), no. 2, 159-174.

[19] E. Hebey and M. Vaugon, From best constants to critical functions, Math. Z. 237 (2001), no. 4, 737-767. 
[20] E. Hebey and J.-C. Wei, Resonant states for the static Klein-Gordon-Maxwell-Proca system, Math. Res. Lett. To appear.

[21] M. A. Khuri, F. C. Marques, and R. M. Schoen, A compactness theorem for the Yamabe problem, J. Differential Geom. 81 (2009), no. 1, 143-196.

[22] J. M. Lee and T. H. Parker, The Yamabe problem, Bull. Amer. Math. Soc. (N.S.) 17 (1987), no. 1, 37-91.

[23] Y.-Y. Li, On a singularly perturbed elliptic equation, Adv. Differential Equations 2 (1997), no. 6, 955-980.

[24] Y.-Y. Li and L. Zhang, A Harnack type inequality for the Yamabe equation in low dimensions, Calc. Var. Partial Differential Equations 20 (2004), no. 2, 133-151.

[25] _ Compactness of solutions to the Yamabe problem. II, Calc. Var. Partial Differential Equations 24 (2005), no. 2, 185-237.

[26] Y.Y. Li and L. Zhang, Compactness of solutions to the Yamabe problem. III, J. Funct. Anal. 245 (2007), no. $2,438-474$.

[27] Y.-Y. Li and M.-J. Zhu, Yamabe type equations on three-dimensional Riemannian manifolds, Commun. Contemp. Math. 1 (1999), no. 1, 1-50.

[28] F. C. Marques, A priori estimates for the Yamabe problem in the non-locally conformally flat case, J. Differential Geom. 71 (2005), no. 2, 315-346.

[29] A. M. Micheletti, A. Pistoia, and J. Vétois, Blow-up solutions for asymptotically critical elliptic equations, Indiana Univ. Math. J. 58 (2009), no. 4, 1719-1746.

[30] M. Musso and A. Pistoia, Multispike solutions for a nonlinear elliptic problem involving the critical Sobolev exponent, Indiana Univ. Math. J. 51 (2002), no. 3, 541-579.

[31] A. Pistoia and G. Vaira, On the Stability for the Paneitz-Branson equation, Int. Math. Res. Notices. doi: 10.1093/imrn/rns133.

[32] A. Pistoia and J. Vétois, Sign-changing bubble towers for asymptotically critical elliptic equations on Riemannian manifolds (2012). Preprint.

[33] S. I. Pohožaev, On the eigenfunctions of the equation $\Delta u+\lambda f(u)=0$, Soviet Math. Dokl. 6 (1965), 1408-1411.

[34] O. Rey, Proof of two conjectures of H. Brézis and L. A. Peletier, Manuscripta Math. 65 (1989), no. 1, $19-37$.

[35] —. The role of the Green's function in a nonlinear elliptic equation involving the critical Sobolev exponent, J. Funct. Anal. 89 (1990), no. 1, 1-52.

[36] F. Robert and J. Vétois, Sign-changing blow-up for scalar curvature type equations, Comm. Partial Differential Equations. To appear.

[37] R. M. Schoen, Conformal deformation of a Riemannian metric to constant scalar curvature, J. Differential Geom. 20 (1984), no. 2, 479-495.

[38] _ Variational theory for the total scalar curvature functional for Riemannian metrics and related topics, Topics in calculus of variations (Montecatini Terme, 1987), Lecture Notes in Math., vol. 1365, Springer, Berlin, 1989, pp. 120-154.

[39] R. M. Schoen and S.-T. Yau, On the proof of the positive mass conjecture in general relativity, Comm. Math. Phys. 65 (1979), no. 1, 45-76.

[40] _ Conformally flat manifolds, Kleinian groups and scalar curvature, Invent. Math. 92 (1988), no. 1, $47-71$.

[41] N. S. Trudinger, Remarks concerning the conformal deformation of Riemannian structures on compact manifolds, Ann. Scuola Norm. Sup. Pisa (3) 22 (1968), 265-274.

[42] H. Yamabe, On a deformation of Riemannian structures on compact manifolds, Osaka Math. J. 12 (1960), $21-37$.

Pierpaolo Esposito, Dipartimento di Matematica, Università degli Studi "Roma Tre", Largo

S. LeOnardo Murialdo 1, 00146 Roma, Italy

E-mail address: esposito@mat.uniroma3.it

Angela Pistoia, Dipartimento SBai, Università di Roma "La Sapienza", via Antonio Scarpa 16, 00161 Roma, ItTALY

E-mail address: pistoia@dmmm.uniroma1.it

Jérôme Vétois, Université de Nice - Sophia Antipolis, Laboratoire J.-A. Dieudonné, UmR CNRS-UnS 7351, Parc Valrose, 06108 Nice Cedex 2, France

E-mail address: vetois@unice.fr 